Estudo de Cinética e Equilíbrio de Adsorção Empregando a Casca do Coco Modificada Quimicamente para a Remoção de $\mathrm{Pb}$ (II) de Banho Sintético

\author{
da Silva, J. E.; Rodrigues, F. I. L.; Pacífico, S. N.; Santiago, L. F.; Muniz, C. \\ R.; Saraiva, G.D.; Nascimento, R. F.; Sousa Neto, V. O*
}

Rev. Virtual Quim., 2018, 10 (5), 1248-1262. Data de publicação na Web: 5 de novembro de 2018

http://rvq.sbq.org.br

\title{
Study of Kinetics and Adsorption Equilibrium Employing Chemically Modified Coconut Shell for the Removal of $\mathrm{Pb}$ (II) from Synthetic Bath
}

Abstract: Currently, the industrial sector that employs lead metal in its production, such as the manufacture of cable linings, building elements, pigments, soft welds, and ammunition has caused great contamination in industrial effluents. Faced with these problems, several methods have been applied to reduce the concentration of this toxic metal. The objective of this work is to obtain a low-cost biosorbent with a capacity to remove $\mathrm{Pb}$ (II) ions. The modification was through a controlled polymerization reaction in sulfuric medium employing formaldehyde as the starting reagent. The Langmuir, Freundlich, and Temkin models adequately described the adsorption process. They indicated that the modified adsorbent removed $\mathrm{Pb}$ (II) with good efficiency and performance. The adsorption capacity of the biosorbent was found to be $120.48 \mathrm{mg}^{-1} \mathrm{~g}^{-1}$ wich is higher or comparable to the adsorption capacity of various adsorbents reported in literature. The adsorption kinetics indicated that equilibrium was reached within $30 \mathrm{~min}$ for the conditions studied and that intrapore diffusion is the limiting step of the $\mathrm{Pb}$ (II) adsorption process. The results indicated that CCFP has the high potential for removal of $\mathrm{Pb}$ (II) in water.

Keywords: Adsorption kinetics; coconut shell; lead.

\section{Resumo}

Atualmente o setor industrial que emprega o metal chumbo na sua produção, tais como, fabricação de forros para cabos, elemento de construção civil, pigmentos, soldas suaves e munições, tem causado grande contaminação em efluentes industriais. Diante desse problema, vários métodos vêm sendo aplicados para reduzir a concentração desse metal tóxico. 0 objetivo deste trabalho é obter um biossorvente de baixo custo com capacidade para remover íons $\mathrm{Pb}(\mathrm{II})$. A modificação foi feita mediante uma reação de polimerização controlada em meio sulfúrico empregando formaldeído como reagente de partida. Os modelos de Langmuir, Freundlich, e Temkin descreveram adequadamente o processo de adsorção. Eles indicaram que o adsorvente modificado removeu o $\mathrm{Pb}$ (II) com boa eficiência e performance. A capacidade de adsorção do biossorvente foi de $120,48 \mathrm{mg} \cdot \mathrm{g}^{-1}$, o que é maior ou comparável à capacidade de adsorção de vários adsorventes relatados na literatura. A cinética de adsorção indicou que o equilíbrio foi alcançado dentro de 30 min para as condições estudadas e que a difusão intraporo é a etapa limitante do processo de adsorção do $\mathrm{Pb}(\mathrm{II})$. Os resultados indicaram que o CCFP possui o alto potencial de remoção de $\mathrm{Pb}(I I)$ na água.

Palavras-chave: Cinética de adsorção; casca do coco; chumbo.

\footnotetext{
* Universidade Estadual do Ceará (UECE), Faculdade de Educação Ciências e Letra do Sertão Central (FECLESC), Departamento de Química, Rua José de Queiroz Pessoa 2554, Planalto Universitário, CEP 63900-000, Quixadá-CE, Brasil

Mvicente.neto@uece.br DOI: $\underline{10.21577 / 1984-6835.20180086}$
} 


\title{
Estudo de Cinética e Equilíbrio de Adsorção Empregando a Casca do Coco Modificada Quimicamente para a Remoção de $\mathrm{Pb}$ (II) de Banho Sintético
}

\author{
José E. da Silva, ${ }^{a}$ Francisco I. L. Rodrigues, ${ }^{a}$ Sara N. Pacífico, ${ }^{a}$ Leonardo F. \\ Santiago, ${ }^{\text {a }}$ Celio R. Muniz, ${ }^{\text {b }}$ Gilberto D. Saraiva, ${ }^{\mathrm{b}}$ Ronaldo F. do \\ Nascimento, ${ }^{c}$ Vicente de O. Sousa Neto ${ }^{\mathrm{b}, *}$ \\ a Universidade Estadual do Ceará (UECE), Departamento de Química, Centro de Educação, \\ Ciências e Tecnologia da Região dos Inhamuns (CECITEC), CEP 63660-000, Tauá-CE, Brasil.

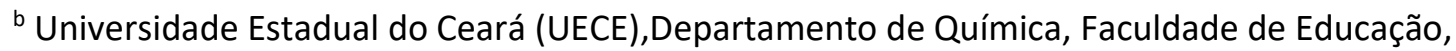 \\ Ciências e Letras do Sertão Central (FECLESC), CEP 63900-000, Quixadá-CE, Brasil. \\ ' Universidade Federal do Ceará, Departamento de Química Analítica e Físico Química Campus \\ do Pici, CEP 60451-970, Fortaleza-CE, Brasil. \\ * vicente.neto@uece.br
}

Recebido em 14 de fevereiro de 2018. Aceito para publicação em 1 de outubro de 2018

1. Introdução

2. Material e Métodos

2.1. Adsorvente

2.2. Solução sintética de $\mathrm{Pb}(\mathrm{II})$

2.3. Tratamento da casca de coco com formaldeído polimerizado (CCFP)

2.4. Estudo de equilíbrio

2.5. Estudo cinético e de mecanismo

2.6. Potencial de carga zero (PZC)

\section{Resultados e Discussão}

3.1. Potencial de carga zero (PZC)

3.2. Estudo de equilíbrio

3.3. Análise de regressão não linear $(\chi 2)$

3.4. Estudo cinético e mecanismo

\section{Conclusão}

\section{Introdução}

A contaminação de águas por metais pesados oriundos de efluentes industriais é um problema sério e preocupante devido à alta toxicidade, abundância e persistência 
destes contaminantes. ${ }^{1,2}$ Outro problema ambiental associado aos metais pesados é a sua subsequente acumulação em habitats aquáticos, microrganismos, na flora e fauna, que, por sua vez, podem entrar na cadeia alimentar e provocar efeitos significativos sobre a saúde humana em longo prazo. ${ }^{2}$

Atualmente tem-se observado uma grande demanda pelo uso industrial do chumbo (mineração, baterias, pinturas, cerâmica). ${ }^{3,4}$ Isso tem afetado também a saúde dos seres humanos, acarretando problemas de diversas naturezas. Em humanos, a acumulação de chumbo no organismo pode afetar severamente as funções cerebrais, sangue, rins, sistema digestivo e reprodutor, inclusive com possibilidade de produzir mutações genéticas em descendentes. ${ }^{5}$

Diante das sérias consequências causadas pela contaminação por $\mathrm{Pb}(\mathrm{II})$, vários métodos tais como adsorção, degradação fotocatalítica, oxidação, membranas nanofiltrantes e eletrólise, ${ }^{6-10}$ vêm sendo desenvolvidos com o objetivo de reduzir os impactos ambientais.

A adsorção é considerada uma das mais competitivas técnicas de remoção de metais pesados contidos em águas residuais industriais. ${ }^{11}$ No entanto, explorar e desenvolver adsorventes com alta capacidade de adsorção e eficiência continua sendo um desafio. ${ }^{11,12}$

O coco é cultivado em mais de 86 países, estando o Brasil entre os maiores produtores do fruto. No entanto, a grande produção e o descarte inadequado também contribuem para a poluição do meio ambiente, produzindo uma grande quantidade de resíduo sólido. ${ }^{13}$ Tendo em vista a grande poluição causada pela casca do coco, muitas pesquisas têm sido direcionadas à remoção de poluentes presentes na água em função de sua afinidade natural por metais. ${ }^{14} \mathrm{~A}$ casca de coco é um resíduo agrícola que pode ser considerada uma alternativa de baixo custo econômico e operacional no tratamento de efluentes industriais. ${ }^{14}$

Neste estudo, três modelos cinéticos de adsorção foram aplicados: pseudo-primeira ordem, pseudo-segunda ordem e o modelo de Elovich. Os modelos foram aplicados na sua forma não linear. No estudo de isoterma de adsorção aplicaram-se os modelos não lineares de Langmuir, Temkin e Freundlich.

O erro associado às funções não lineares foi calculado a partir da função erro Quiquadrado $\left(\mathrm{c}^{2}\right)$ usando o suplemento Solver da planilha da Microsoft, Excel 2013. O estudo de mecanismo de difusão foi feito aplicando-se os modelos de Webber Morris e de Boyd.

O objetivo do trabalho é obter um bioadsorvente de baixo custo por meio do estudo de cinética e isoterma de adsorção, aplicando a casca do coco modificada com formaldeído polimerizado para remoção de $\mathrm{Pb}(\mathrm{II})$.

\section{Material e Métodos}

\subsection{Adsorvente}

O coco foi obtido no município de AiuabaCeará. A casca foi exposta ao sol para secagem por 24h, cortada em pequenos pedaços, triturada e peneirada para separação da fração 60-100 mesh. As frações foram secas a $60{ }^{\circ} \mathrm{C}$ por $24 \mathrm{~h}$ em estufa. ${ }^{15}$

\subsection{Solução sintética de $\mathrm{Pb}(\mathrm{II})$}

Para esse estudo foram preparadas soluções sintéticas de $\mathrm{Pb}(\mathrm{II})$ com concentrações de 100, 300 e $500 \mathrm{mg} . \mathrm{L}^{-1}$, utilizando o reagente nitrato de chumbo $\left(\mathrm{PbNO}_{3}\right), \mathrm{pH}$ 5,5 em tampão acetato.

\subsection{Tratamento da casca de coco com formaldeído polimerizado (CCFP)}

A casca de coco bruta ("in natura") foi tratada com ácido sulfúrico $\left(\mathrm{H}_{2} \mathrm{SO}_{4}\right)$ e formaldeído $(\mathrm{HCHO}) 37 \% .{ }^{16}$ A mistura foi aquecida a $60^{\circ} \mathrm{C}$ por $5 \mathrm{~h}$ sob agitação contínua. As amostras CCFP obtidas foram filtradas e 
lavadas repetidas vezes com água destilada, a fim de se retirar o excesso do reagente, e seca em estufa a $60{ }^{\circ} \mathrm{C}$ por 24 horas..$^{15}$

\subsection{Estudo de equilíbrio}

O estudo de equilíbrio de adsorção foi executado, inicialmente, agitando-se os frascos cônicos de $100 \mathrm{~mL}$ contendo $50 \mathrm{mg}$ do adsorvente (CCFP) com $25 \mathrm{~mL}$ de solução de $\mathrm{Pb}(\mathrm{II})$, na faixa de concentração de 50 a 500 $\mathrm{mg} . \mathrm{L}^{-1}$. A agitação foi mantida em $150 \mathrm{rpm}$ em um agitador orbital. A temperatura foi mantida a $25{ }^{\circ} \mathrm{C}$. O tempo de agitação foi fixado em 120 minutos, e o $\mathrm{pH}$ igual a 5,5 foi mantido com tampão acético.

As concentrações antes e depois do estudo de equilíbrio foram determinadas por titulação complexiométrica, empregando uma

$$
\mathrm{qt}=\frac{(\mathrm{Co}-\mathrm{Ct}) \cdot \mathrm{V}}{\mathrm{m}}
$$

Em que: Ct (mg. $\left.\mathrm{L}^{-1}\right)$ é a concentração da fase líquida de $\mathrm{Pb}(\mathrm{II})$, no tempo $\mathrm{t}$ (min), Co (mg. $\mathrm{L}^{-1}$ ) representa a concentração inicial da solução do metal; $\mathrm{V}(\mathrm{L})$. V corresponde à alíquota da solução de metal empregada (em litros).

\subsection{Potencial de carga zero (PZC)}

O instrumento Zetasizer Nano ZS (Malvern, GBR) foi usado para medir o potencial zero de todas as amostras.

\section{Resultados e Discussão}

\subsection{Potencial de carga zero (PZC)}

solução padrão de EDTA. As capacidades de adsorção foram calculadas com base na Equação (1).

\subsection{Estudo cinético e de mecanismo}

O estudo cinético foi realizado mediante a retirada das amostras do banho de equilíbrio em intervalos de tempo de 2, 4, 6, 8, 10, 15, $20,30,40$ e 50 minutos. A massa do adsorvente usada foi de $50 \mathrm{mg}$ em contato com $25 \mathrm{~mL}$ de solução sintética de $\mathrm{Pb}(\mathrm{II})$. $\mathrm{O}$ residual de $\mathrm{Pb}(\mathrm{II})$ e a concentração total, em cada estudo cinético, foi quantificado da mesma forma que no estudo de adsorção. Após cada tempo, as amostras com o adsorvente foram filtradas e armazenadas para a titulação. A capacidade de adsorção foi calculada de acordo com as concentrações do metal e foram determinadas pela Equação (1).

$\mathrm{O}$ pH afeta a adsorção a medida em que determina o grau de distribuição das espécies químicas que devem ser removidas e por influenciar na distribuição de cargas na superfície do adsorvente. O PZC, potencial de carga zero, é uma medida conveniente da tendência de uma superfície se tornar positiva ou negativamente carregada em função do $\mathrm{pH}$. É o valor requerido para que a carga líquida do adsorvente seja nula, o chamado ponto de carga zero ( $\mathrm{pH}_{\mathrm{PZC}}$ ). Para valores de $\mathrm{pH}$ inferiores ao $\left(\mathrm{pH}_{\mathrm{PzC}}\right)$, a carga superficial é positiva e a adsorção de ânions é favorecida e para valores de $\mathrm{pH}$ superiores ao $\left(\mathrm{pH}_{\mathrm{PzC}}\right)$ a carga superficial é negativa e a adsorção de cátions é favorecida. ${ }^{17}$ Na Figura 1 é mostrado o potencial de carga zero para a casca do coco bruta (CCB) e modificada (CCFP) em função do $\mathrm{pH}$. 


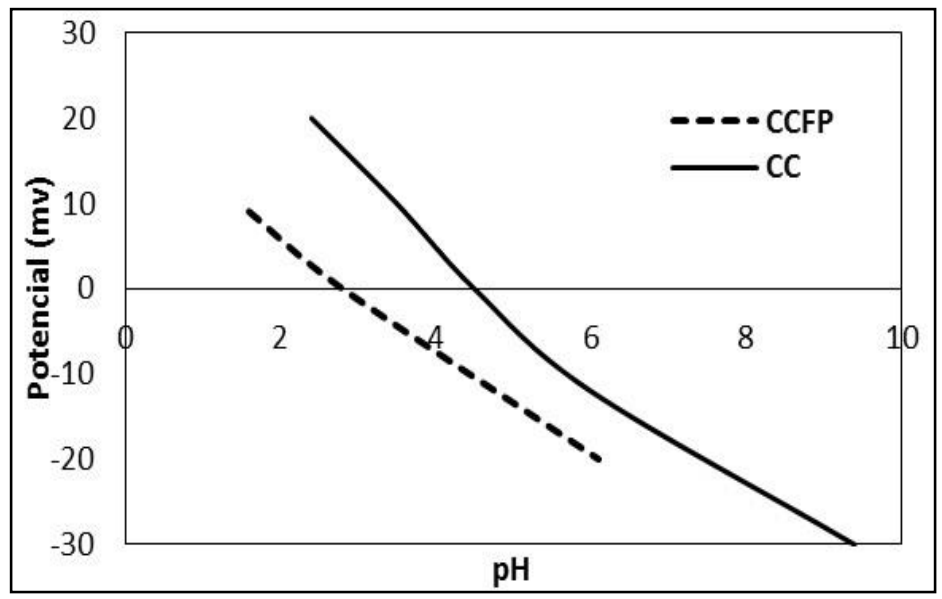

Figura 1. Ponto de carga zero (PZC) da casca de coco bruto (CCB) e casca de coco polimerizado (CCFP)

\subsection{Estudo de Equilíbrio}

A condição de equilíbrio é importante, pois permite que seja feito a determinação experimental da capacidade máxima de adsorção em condições controladas. A capacidade de adsorção q $\left(\mathrm{mg} \cdot \mathrm{g}^{-1}\right)$ é a relação entre a quantidade que é adsorvida de uma determinada espécie química, por unidade de massa do adsorvente. No estudo de equilíbrio estuda-se, à temperatura constante, o efeito da concentração da espécie química na

$$
\mathrm{q}_{\mathrm{e}}=\frac{\mathrm{q}_{\max \cdot \mathrm{K}_{\mathrm{L}} \cdot \mathrm{C}_{\mathrm{e}}}}{\left(1+\mathrm{K}_{\mathrm{L}} \cdot \mathrm{C}_{\mathrm{e}}\right)}
$$

Em que: $q_{e}\left(m g \cdot g^{-1}\right)$ e $C_{e}\left(m g . L^{-1}\right)$ são a quantidade de material adsorvido por unidade de massa do adsorvente e concentração de metal em solução (mantida em condições que se atinja o equilíbrio), respectivamente, $q_{\max }$ (mg. $\mathrm{g}^{-1}$ ) é a quantidade máxima do metal adsorvido por unidade de massa de adsorvente.

Na Tabela 1 é mostrado a capacidade máxima de adsorção $q_{\max }\left(\mathrm{mg} \mathrm{g}^{-1}\right)$ e a constante de Langmuir $\mathrm{K}_{\mathrm{L}}\left(\mathrm{L}_{\mathrm{mg}}{ }^{-1}\right)$ para o adsorvente CCFP, os valores encontrados foram 120,48 mg.g ${ }^{-1}$ e $1,59 \times 10^{-2}$ L.mg. ${ }^{-1}$, respectivamente. Na Figura 2 é mostrada a isoterma de adsorção para a remoção de $\mathrm{Pb}$ (II) com CCFP. Observa-se que os dados capacidade de adsorção mantendo-se constante a massa do adsorvente.

\subsubsection{Isoterma de Langmuir}

$\mathrm{O}$ modelo de Langmuir $^{18}$ sugere que a adsorção ocorre em uma superfície homogênea, formando monocamada, sem interação entre espécies adsorvidas. Este modelo é descrito na forma não linear pela Equação (2).

teóricos do modelo de Langmuir e os experimentais são concordantes em todas as faixas de concentrações estudadas.

\subsubsection{Isoterma de Freundlich}

A equação referente à isoterma de equilíbrio de Freundlich é uma equação empírica usada para a descrição da adsorção. O modelo sugere que a adsorção ocorre com formação de multicamadas (energias de adsorção diferentes) com interação entre moléculas adsorvidas. ${ }^{19}$ O modelo de Freundlich é expresso pela Equação (3). 


$$
\mathrm{qe}=\mathrm{K}_{\mathrm{F}} \cdot \mathrm{C}_{\mathrm{e}}{ }^{1 / \mathrm{n}}
$$

Em que: $q_{e}\left(m g \cdot g^{-1}\right)$ é a quantidade de equilíbrio adsorvida, $C_{e}\left(m g . \mathrm{L}^{-1}\right)$ é a concentração de equilíbrio do adsorvato. $\mathrm{K}_{\mathrm{F}} \mathrm{e}$ $\mathrm{n}$ são constantes de Freundlich relacionadas à capacidade de adsorção e o coeficiente de afinidade, respectivamente.

Os parâmetros $K_{F}$ e $n$ são mostrados na Tabela $1 \mathrm{com}$ valores iguais a 17,33 e 3,25, respectivamente. De acordo com a literatura, ${ }^{20} \mathrm{em}$ média, a adsorção favorável tende a ter o valor de $n$ entre 1 e 10 . O valor encontrado para $\mathrm{n}$ sugere que há afinidade do íon $\mathrm{Pb}$ (II) pela superfície do adsorvente CCFP. Os dados se ajustaram bem ao modelo de

$$
\theta=\frac{\mathrm{RT}}{\Delta \mathrm{Q}} \ln \mathrm{K}_{0} \cdot \mathrm{C}_{\mathrm{e}}
$$

Em que: $\left(q_{e} / q_{m}\right)$ é a cobertura fracionada; $\mathrm{k}_{\mathrm{T}}\left(\mathrm{L} \cdot \mathrm{mg}^{-1}\right)$ é a constante de equilíbrio de Temkin; R (J.K $\left.{ }^{-1}\right)$ constante dos gases; $\mathrm{T}(\mathrm{K})$ é a temperatura absoluta e $\Delta \mathrm{Q}=(-\Delta \mathrm{H})\left(\mathrm{kJ} \cdot \mathrm{mol}^{-1}\right)$ é a variação da energia de adsorção.

De acordo com o modelo de Temkin, os parâmetros (Tabela 1), energia de adsorção $(\Delta \mathrm{H})$ e constante de Temkin $\mathrm{k}_{\mathrm{T}}$ assumiram
Freundlich que pode ser observado na Figura (2).

\subsubsection{Isoterma de Temkin}

A isoterma de Temkin assume que o calor de adsorção de todas as moléculas adsorvidas diminui linearmente com a cobertura devido à interação adsorvente-adsorvato, e que a adsorção é caracterizada por uma distribuição uniforme de energias de ligação. ${ }^{21} \mathrm{O}$ modelo de Temkin é definido pela Equação (4).

valores de $-15,77 \mathrm{~kJ} \cdot \mathrm{mol}^{-1}$ e 0,75 L.mg ${ }^{-1}$, respectivamente.

$\mathrm{O}$ valor de $\Delta \mathrm{H}$ negativo sugere que a adsorção de $\mathrm{Pb}$ (II) no adsorvente CCFP, ocorreu por um processo exotérmico.

A Figura (2) ilustra os dados teóricos que têm uma boa concordância com os dados experimentais.

Tabela 1. Parâmetros dos modelos não-lineares de Langmuir, Freundlich e Temkin, para a adsorção de $\mathrm{Pb}$ (II) no adsorvente CCFP

\begin{tabular}{lll}
\hline \multicolumn{1}{c}{ Modelos } & \multicolumn{1}{c}{ Parâmetros } \\
\hline Langmuir & $\mathrm{Q}_{\text {max. }}\left(\mathrm{mg} \cdot \mathrm{g}^{-1}\right)$ & 120,48 \\
& $\mathrm{k}_{\mathrm{L}}\left(\mathrm{mg} \cdot \mathrm{L}^{-1}\right)$ & $1,59 \times 10^{-2}$ \\
& $\chi^{2}$ & 0,72 \\
Freundlich & $\mathrm{N}$ & 3,25 \\
& $\mathrm{k}_{\mathrm{F}}\left(\left(\mathrm{mg} \cdot \mathrm{g}^{-1}\right)\left(\mathrm{L} \cdot \mathrm{mg}^{-1}\right)^{1 / \mathrm{n}}\right)$ & 17,33 \\
& $\chi^{2}$ & 0,18 \\
\multirow{2}{*}{ Temkin } & $\Delta \mathrm{H}\left(\mathrm{kJ} \cdot \mathrm{mol}^{-1}\right)$ & $-15,77$ \\
& $\mathrm{k}_{\mathrm{T}}\left(\mathrm{L} \cdot \mathrm{mg}^{-1}\right)$ & 0,39 \\
& $\chi^{2}$ & 1,06 \\
\hline
\end{tabular}




\subsection{Análise de regressão não linear $\left(\chi^{2}\right)$}

A função estatística Qui-quadrado $\left(\chi^{2}\right)$, é basicamente, a soma dos quadrados das diferenças entre os dados experimentais e os dados previstos teoricamente dos modelos. Assim, se os dados de modelo foram semelhantes aos dados experimentais, $\chi^{2}$ (Equação 5) será um número pequeno e viceversa.

$$
\chi^{2}=\sum \frac{\left(\mathrm{q}_{\mathrm{exp}}-\mathrm{q}_{\mathrm{cal}}\right)^{2}}{\mathrm{q}_{\mathrm{cal}}}
$$

Com base na Equação 5 foram calculados os valores da função erro $\chi^{2}$, que estão representados na Tabela 1 . Os valores de $\chi^{2}$ para Langmuir $\left(\chi^{2}=0,78\right)$, Freundlich $\left(\chi^{2}=\right.$ $0,18)$ e Temkin $\left(\chi^{2}=1,06\right)$ sugerem que os modelos apresentaram baixos erros na descrição do fenômeno, o que explica a boa concordância entre dados experimentais e teóricos. $\mathrm{Na}$ Figura 2 é mostrada a comparação entre os dados teóricos e experimentais para os três modelos de isotermas, aplicados no estudo de equilíbrio. Observa-se a boa concordância entre os resultados.

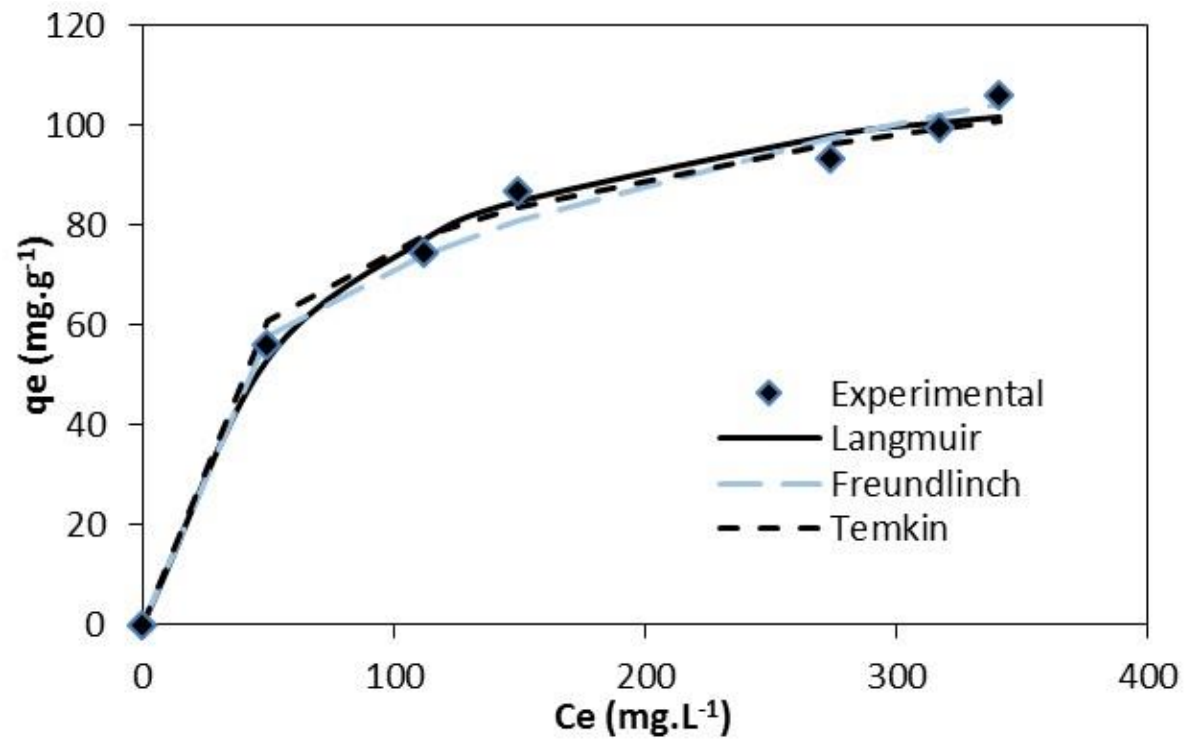

Figura 2. Isoterma de adsorção de $\mathrm{Pb}(\mathrm{II})$ sobre a CCFP, empregando regressão não linear dos modelos de Langmuir, Temkin e Freundlich. Condições: dose de adsorvente 2 g. L $^{-1}$; velocidade de agitação $150 \mathrm{rpm} ; \mathrm{pH}=5,5$; Temperatura $30^{\circ} \mathrm{C}$

\subsection{Estudo cinético e mecanismo}

3.4.1 Efeito da concentração inicial do adsorvato

A Figura 3 ilustra o efeito do tempo de contato sobre a adsorção em batelada com amostras em concentrações iniciais de 100 ,
300 e 500 mg. $\mathrm{L}^{-1}$ de $\mathrm{Pb}(\mathrm{II})$ a $25{ }^{\circ} \mathrm{C}$. A quantidade de adsorção aumenta com o tempo na fase inicial. (0-10 $\mathrm{min})$, até atingir um tempo de equilíbrio de aproximadamente $30 \mathrm{~min}$. A partir do tempo de equilíbrio o aumento adicional no tempo de contato teve um efeito insignificante sobre a quantidade de adsorção. A adsorção rápida em diferentes concentrações de $\mathrm{Pb}$ (II) nos primeiros minutos (<2 $\mathrm{min}$ ) pode ser atribuída à disponibilidade 
de um grande número de sítios disponíveis no CCFP no estágio inicial da adsorção. A taxa de adsorção diminui gradualmente durante o processo de adsorção até atingir o equilíbrio.

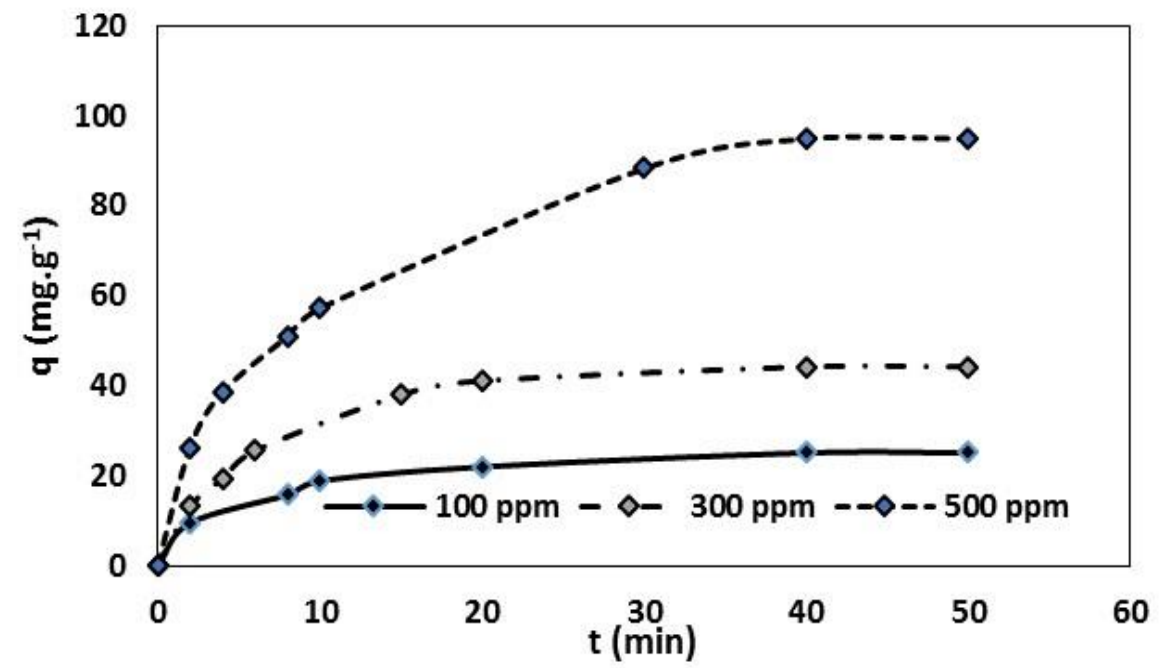

Figura 3. Avaliação da quantidade de adsorção de $\mathrm{Pb}(\mathrm{II})$ com o tempo, para diferentes concentrações. Condições: dose de adsorvente $2 \mathrm{~g}^{\circ} \mathrm{L}^{-1}$; Velocidade $150 \mathrm{rpm} ; \mathrm{pH}=5,5$;

Temperatura $30^{\circ} \mathrm{C}$

\subsubsection{Modelos cinéticos}

O estudo cinético de adsorção de $\mathrm{Pb}(\mathrm{II})$ em CCFP utilizou os modelos cinéticos de pseudoprimeira ordem, pseudo-segunda ordem e equação de Elovich. A determinação dos parâmetros foi obtida por meio de regressão não linear. Os resultados estão listados na Tabela 2.

\subsubsection{Pseudo-primeira ordem}

Este modelo avalia se a cinética de adsorção dos íons metálicos é prioritariamente controlada por difusão externa, e independe da concentração do adsorvato. $^{22}$ É definido devidamente pela forma não linear expressa na Equação (6).

$$
\mathrm{qt}=\mathrm{q}_{\mathrm{e}}\left(1-\mathrm{e}^{-\mathrm{k}_{1} \mathrm{t}}\right)
$$

Em que: $\mathrm{q}_{\mathrm{e}}\left(\mathrm{mg}^{\mathrm{g}} \mathrm{g}^{-1}\right)$ é a quantidade adsorvida na fase sólida no equilíbrio; $q(t)$ $\left(\mathrm{mg} \cdot \mathrm{g}^{-1}\right)$ quantidade de metal adsorvido no tempo $t ; \mathrm{t}(\mathrm{min})$ tempo; $\mathrm{k}_{1}\left(\mathrm{~min}^{-1}\right)$ constante de velocidade de pseudo-primeira ordem.

As constantes de velocidade de primeira ordem $\mathrm{k}_{1}$ para 100,300 e $500 \mathrm{mg}^{-\mathrm{L}^{-1}}$ de $\mathrm{Pb}$ (II) foram $1,2 \times 10^{-1}, 9,7 \times 10^{-2}$ e $1,3 \times 10^{-1}, \mathrm{~min}^{-1}$ respectivamente, os valores estão na Tabela 2.

$$
\mathrm{q}_{\mathrm{t}}=\frac{\mathrm{k}_{2} \mathrm{q}_{\mathrm{e}}^{2} \mathrm{t}}{1+\mathrm{k}_{2} \mathrm{q}_{\mathrm{e}} \mathrm{t}}
$$

\subsubsection{Pseudo-segunda ordem}

A taxa de adsorção no modelo de pseudosegunda ordem é dependente da quantidade da espécie química adsorvida na superfície do adsorvente e a quantidade adsorvida no estado de equilíbrio. ${ }^{23} \mathrm{O}$ modelo de pseudosegunda ordem pode ser representado pela Equação (7). 
Em que: $k_{2}$ (g.mg. $\mathrm{min}^{-1}$ ) é a constante de velocidade de modelo de pseudo-segunda ordem.

$\mathrm{Na}$ tabela 2 são listados os valores de $\mathrm{k}_{2}$ para as concentrações de 100, 300 e $500 \mathrm{mg} . \mathrm{L}^{-}$ ${ }^{1}$ com valores $9,15 \times 10^{-3} ; 3,3 \times 10^{-3} ; 1,21 \times 10^{-2}$ g. $\mathrm{mg}^{-1} \cdot \mathrm{min}^{-1}$ respectivamente, $\mathrm{Na}$ mesma tabela é mostrado os valores de qe iguais a 26,$77 ; 51,38 ; 109,08 \mathrm{mg} \cdot \mathrm{g}^{-1}$ respectivamente. Os valores de $\chi^{2}$ iguais a $0,31,0,25$, e 1,48 mg. $\mathrm{L}^{-1}$ respectivamente, mostrando que o modelo de segunda ordem apresentou menores valores de $\chi^{2}$, indicando assim menor erro. Comprovando que o modelo de pseudosegunda se ajustou melhor em comparação ao modelo de primeira ordem. A curva de adsorção para o modelo de pseudo-segunda ordem é mostrada na Figura (4).

\subsubsection{Equação de Elovich}

A equação de Elovich assume que as superfícies sólidas reais são energeticamente heterogêneo e que nem dessorção nem interações entre as espécies adsorvidas podem afetar substancialmente a cinética de adsorção em baixa cobertura de superfície. 0 efeito crucial da heterogeneidade de energia na superfície em equilíbrio de adsorção nos sistemas de gás / sólido. ${ }^{24} \mathrm{~A}$ equação não linear de Elovich é expressada de acordo com a Equação (8).

Em que: $\alpha\left(\mathrm{mg} \mathrm{g}^{-1} \mathrm{~min}^{-1}\right)$ é a taxa de adsorção inicial e $\beta\left(\mathrm{mg}^{\left.-g^{-1}\right)}\right.$ representa a constante de dessorção.

Na tabela 2 estão disponíveis os valores correspondentes a taxa de adsorção inicial para 100, 200 e $300 \mathrm{mg}^{-L^{-1}}$ de 17,52, 14,00, 21,37 $\mathrm{mg} \cdot \mathrm{g}^{-1} \cdot \mathrm{min}^{-1}$ respectivamente e da constante de dessorção $\beta$ iguais a $0,22,1,20$, $0,41 \mathrm{mg} \cdot \mathrm{g}^{-1}$ respectivamente, revelando valores altos de $\alpha$ e baixos valores de $\beta$.

É possível observar na Figura (4) a boa concordância dos resultados teóricos do modelo de Elovich com os dados experimentais, isso sugere que o processo envolve uma quimissorção.

$$
\mathrm{q}_{\mathrm{t}}=\frac{1}{\beta} \ln (1+\alpha \beta \mathrm{t})
$$




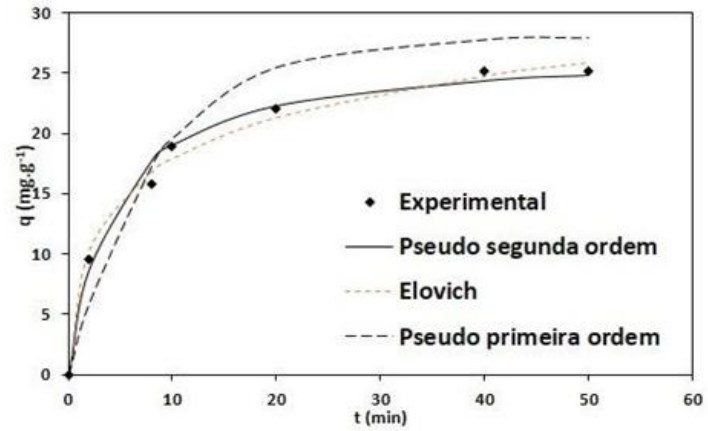

(a)

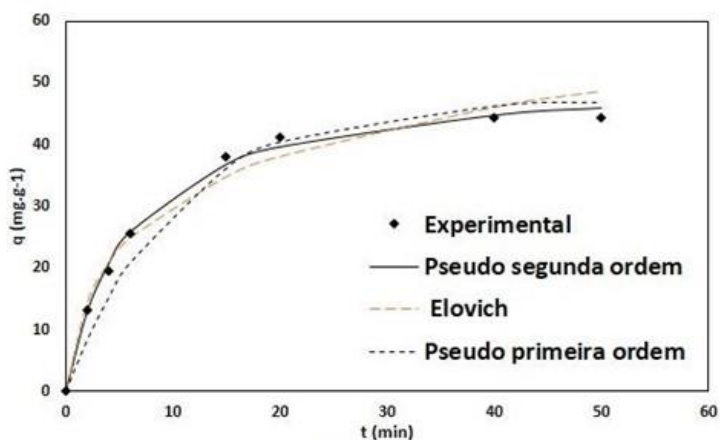

(b)

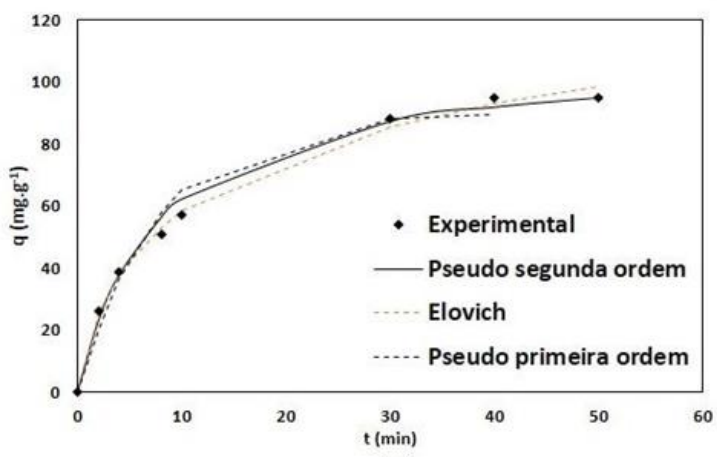

(c)

Figura 4. Cinética de adsorção de íons $\mathrm{Pb}$ (II) no adsorvente CCFP. Condições experimentais: Concentração do íon $\mathrm{Pb}$ (II) (a) $100 \mathrm{mg} \cdot \mathrm{L}^{-1}$ de Pb(II), (b) $300 \mathrm{mg} \cdot \mathrm{L}^{-1} \mathrm{~Pb}$ (II), (c) $500 \mathrm{mg} \cdot \mathrm{L}^{-1}$ de Pb(II). Dosagem de adsorvente: $2 \mathrm{~g} \cdot \mathrm{L}^{-1}$

Tabela 2. Comparação dos parâmetros não lineares de pseudo-primeira ordem, pseudosegunda ordem, e equação de Elovich e de qe calculados e experimentais

\begin{tabular}{|c|c|c|c|}
\hline Modelos & $100 \mathrm{mg} \cdot \mathrm{L}^{-1}$ & $300 \mathrm{mg} \cdot \mathrm{L}^{-1}$ & $500 \mathrm{mg} \cdot \mathrm{L}^{-1}$ \\
\hline \multicolumn{4}{|c|}{ Pseudo primeira ordem } \\
\hline qe (exp) & 28,01 & 47,11 & 95 \\
\hline qe $\left(m g \cdot g^{-1}\right)$ & 25,15 & 44,33 & 90,14 \\
\hline$K_{1}\left(\min ^{-1}\right)$ & 0,12 & $9,7 \times 10^{-2}$ & 0,13 \\
\hline$\chi^{2}$ & 3,31 & 5,71 & 3,88 \\
\hline \multicolumn{4}{|c|}{ Pseudo segunda ordem } \\
\hline qe (exp) & 25,15 & 51,38 & 109,08 \\
\hline qe $\left(m g \cdot g^{-1}\right)$ & 26,77 & 44,33 & 95,00 \\
\hline $\mathrm{K}_{2}\left(\mathrm{~g} \cdot \mathrm{mg}^{-1} \cdot \mathrm{min}^{-1}\right)$ & $9,15 \times 10^{-3}$ & $3,3 \times 10^{-3}$ & $1,21 \times 10^{-2}$ \\
\hline$\chi^{2}$ & 0,31 & 0,25 & 1,48 \\
\hline \multicolumn{4}{|c|}{ Equação de Elovich } \\
\hline$\alpha\left(\mathrm{mg} \cdot g \cdot \min ^{-1}\right)$ & 17,52 & 14,00 & 21,37 \\
\hline$\beta\left(\mathrm{mg} \cdot \mathrm{g}^{-1}\right)$ & 0,20 & 0,084 & 0,037 \\
\hline$\underline{\chi}^{2}$ & 0,22 & 1,22 & 0,41 \\
\hline
\end{tabular}




\subsubsection{Estudo de mecanismo}

O mecanismo de adsorção para a remoção de qualquer espécie química pode-se envolver as seguintes etapas: (a) difusão intrafilme através da camada do solvente que envolve o adsorvente, (b) difusão intraporo, e (c) adsorção da espécie química na superfície do adsorvente. ${ }^{15}$

$$
\mathrm{qt}=\mathrm{kdt}^{1 / 2}+\mathrm{C}
$$

Em que: qt $\left(\mathrm{mg}^{\mathrm{g}} \mathrm{g}^{-1}\right)$ é a quantidade de espécies químicas adsorvidas no momento $\mathrm{t} ; \mathrm{t}$ (min) é o tempo; $k d\left(m g \cdot g^{-1} \cdot \mathrm{min}^{-1 / 2}\right)$ é o coeficiente de difusão no sólido.

$O$ valor de $C$ fornece informações sobre a espessura do filme de solvente que envolve o adsorvente. Em geral, quanto maior for o valor de C (coeficiente linear), maior será a espessura do filme e por consequência maior será seu efeito na taxa de adsorção. Diz-se, neste caso, que o mecanismo de adsorção é controlado pela difusão intrafilme. Quando C

$$
\mathrm{k}_{\mathrm{d}}=\frac{6 \mathrm{q}_{\mathrm{e}}}{\mathrm{R}} \sqrt{\frac{\mathrm{D}}{\pi}}
$$

Tabela 3. Valores do coeficiente de difusão de poros, Constante de difusão, coeficiente de determinação para o modelo de Webber-Morris para diferentes concentrações de $\mathrm{Pb}$ (II)

\begin{tabular}{ccccc}
\hline $\begin{array}{c}\mathrm{Co} \\
\left(\mathrm{mg} \cdot \mathrm{L}^{-1}\right)\end{array}$ & $\begin{array}{c}\mathrm{K}_{\mathrm{d}} \\
\left(\mathrm{mg} \cdot \mathrm{g} \cdot \mathrm{min}^{-1 / 2}\right)\end{array}$ & $\begin{array}{c}\mathrm{C} \\
\left(\mathrm{mg} \cdot \mathrm{g}^{-1}\right)\end{array}$ & $\mathrm{R}^{2}$ & $\begin{array}{c}\mathrm{D} \\
\left(\mathrm{cm}^{2} \cdot \mathrm{s}^{-1}\right)\end{array}$ \\
\hline 100 & 4,14 & 4,31 & 0.961 & $2,96 \times 10^{-6}$ \\
300 & 9,23 & 1,31 & 0.987 & $4,74 \times 10^{-6}$ \\
500 & 17,33 & 2,57 & 0.995 & $3,64 \times 10^{-6}$ \\
\hline
\end{tabular}

Em que: $R(\mathrm{~cm})$ é o raio da partícula; $D$ $\left(\mathrm{cm}^{2} \cdot \mathrm{min}^{-1}\right)$ é o coeficiente de difusão e qe $\left(m g \cdot g^{-1}\right)$ é a concentração da fase sólida em equilíbrio.

A Figura 5 é um gráfico da função de $t^{1 / 2}$ versus qt que confirma a validade da relação

\subsubsection{Modelo de Webber Morris}

De acordo com Weber e Morris, ${ }^{25}$ se a difusão intrafilme é o fator determinante da taxa de adsorção, a remoção do adsorvato varia com a raiz quadrada do tempo como mostrado na Equação (9).

tem valores próximos ou igual a zero diz-se que o mecanismo de adsorção é controlado pela difusão intraporo (ou seja, a porosidade da superfície é determinante na adsorção). Se a difusão intraporo é controladora, então qt versus $\mathrm{t}^{1 / 2}$ será linear, e a reta passará pela origem ou vizinhança. ${ }^{25}$

Na Equação (10), ${ }^{13} \mathrm{k}_{d}$ (mg.g.min ${ }^{-1 / 2}$ ) é definida como taxa de difusão e está relacionada com o coeficiente de difusão (D) pela Equação (10). linear, mostrando que para todas as concentrações estudadas a extrapolação da reta não passou próxima a origem $(C \neq 0$, ver tabela 3).

$O$ resultado sugere a formação de uma película, indicando que a difusão intrafilme é 
o mecanismo controlador do processo de adsorção. Considerando 100, 300 e 500 mg.t. ${ }^{-1}$ de $\mathrm{Pb}(\mathrm{II})$ e aplicando o modelo de Webber Morris na primeira etapa, os valores de $\mathrm{K}_{\mathrm{d}}$ foram de 4,14, 9,23, 17,33 mg.g.min ${ }^{-1 / 2}$ respectivamente. Na tabela 3 são listados os parâmetros do modelo de Weber-Morris e os valores da constante de difusão D. obtidos de (10). De acordo com os resultados, para as três concentrações estudas, verificou-se que para tempos acima de 1 minuto predominou a difusão infrafilme no processo de adsorção de $\mathrm{Pb}$ (II) sobre a superfície do adsorvente CCFP.

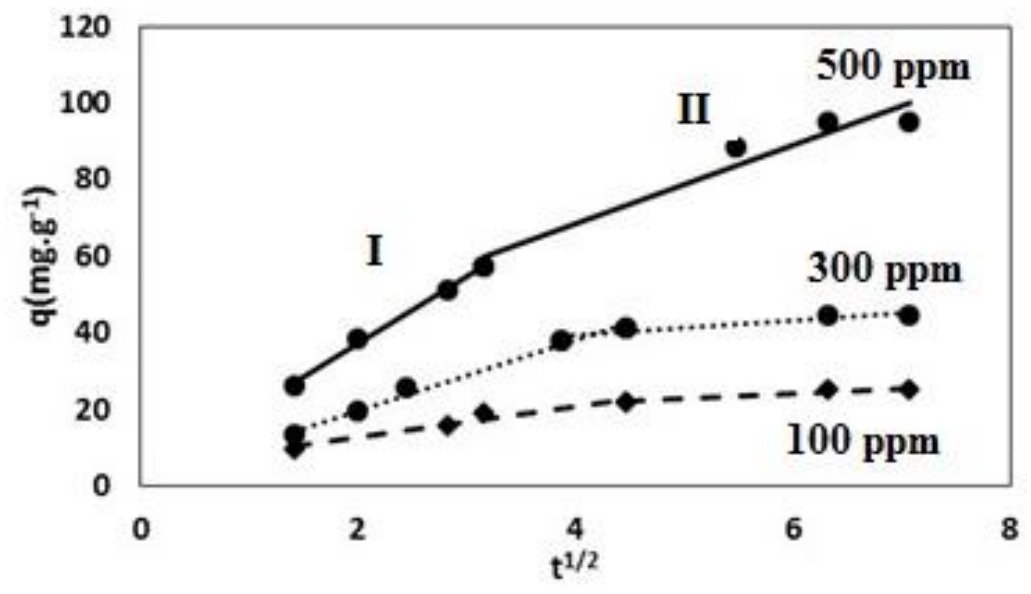

Figura 5. Gráfico do modelo de Weber-Morris para a adsorção de $\mathrm{Pb}(\mathrm{II})$ em CCFP para a concentração inicial de 100,300 e $500 \mathrm{mg}^{-\mathrm{L}^{-1}}$. Dose de adsorvente $=2 \mathrm{~g} \mathrm{~L}^{-1}$

\subsubsection{Modelo de Boyd}

O modelo de Webber-Morris ${ }^{26}$ não permite, com precisão, que se saiba o que ocorre nas proximidades de $\mathrm{t}=0$, pois a equação envolve $t^{1 / 2}$. Desta forma não é possível estimar se nos estágios iniciais (abaixo de 1 minuto no gráfico) predomina ou não a difusão intraporo. Então para confirmar o tipo de difusão nas imediações de $t=0$ os dados cinéticos foram investigados pelo modelo de Boyd $^{27}$ que assume que se a difusão dentro dos poros for a etapa limitante da taxa de adsorção, então os resultados podem ser expressos de acordo com a Equação 11.

Em que: $B$ é uma constante, e $F$ é a capacidade fracional do equilíbrio em tempos diferentes dado pela Equação 12.

$$
\begin{aligned}
& F=1-\left(\frac{6}{\pi^{2}}\right) \sum_{n=1}^{\infty}\left(\frac{1}{n^{2}}\right) e^{\left(-\pi^{2} B t\right)} \\
& F=\frac{q t}{q e}
\end{aligned}
$$

m que: qe e qt são, respectivamente, as capacidades de adsorção no equilíbrio e no tempo $\mathrm{t}$ em $\left(\mathrm{mg} \cdot \mathrm{g}^{-1}\right)$. O termo Bt é calculado pelas seguintes equações de Reichenberg. ${ }^{28}$
O coeficiente de difusão $D\left(\mathrm{~cm}^{2} \cdot \mathrm{min}^{-1}\right)$ está relacionado à constante de Boyd através da Equação (15). 
da Silva, J. E. et al.

$$
\begin{aligned}
& \text { Para } \mathrm{F}>0,85 \quad \mathrm{Bt}=-0.4977-\ln (1-\mathrm{F}) \\
& \text { Para } \mathrm{F}<0,85, \quad \mathrm{Bt}=\left(\sqrt{\pi}-\sqrt{\pi-\left(\frac{\pi^{2} \mathrm{~F}}{3}\right)}\right. \\
& B=\frac{\pi^{2} D}{d^{2}}
\end{aligned}
$$

Em que: $D$ é coeficiente de difusão $\left(\mathrm{cm}^{2} \cdot \mathrm{min}^{-1}\right) ; \mathrm{d}$ é o diâmetro médio das partículas $(\mathrm{cm})$ e B é a constante de Boyd.

Para aplicar este modelo, o termo Bt do produto é calculado para cada valor de F, em

Seguida, os valores $\mathrm{Bt}$ resultantes são plotados versus o tempo (t). Se o gráfico for linear, então a inclinação é igual a B. Uma interceptação em zero do segmento linear que começa de $\mathrm{t}=0$ implicaria que a difusão intraporo é quem controla a taxa de adsorção durante todo o tempo de adsorção.
Aplicando (15) foi obtido as constantes de difusão $2,23 \times 10^{-4}, 1,73 \times 10^{-4}$ e $1,04 \times 10^{-4}$ para as concentrações 500,300 e $100 \mathrm{mg} . \mathrm{L}^{-1}$. Na figura 6 é mostrado o gráfico de Boyd para a adsorção de íon $\mathrm{Pb}$ (II) no CCFP. O gráfico mostra que nas imediações de $\mathrm{t}=0$ ( $\mathrm{t}<1 \mathrm{~min}$ ) a adsorção é controlada por uma difusão intraporo, pois o coeficiente linear das equações da reta aproxima-se de zero para todas as concentrações estudadas.

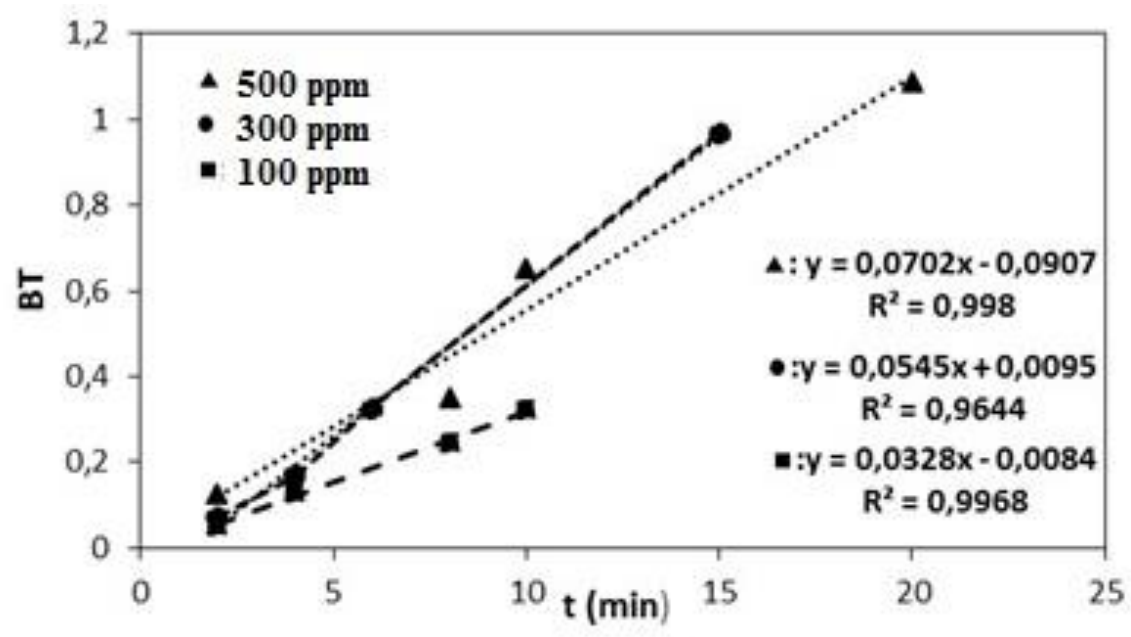

Figura 6. Gráfico do modelo de Boyd para adsorção de 100, 300 e $500 \mathrm{mg}^{-\mathrm{L}^{-1}}$ de $\mathrm{Pb}$ (II) em CCFP. Para F>0,85 aplicado pela Equação (13) e para $F<0,85$ aplicado pela Equação (14)

\section{Conclusão}

Verificou-se que a remoção de $\mathrm{Pb}(I I)$ de solução aquosa por adsorvente modificado CCFP foi eficaz. O estudo de equilíbrio mostrou que os modelos de Langmuir, Freundlich, e Temkin descreveram bem o processo de adsorção. A cinética de adsorção indicou que o equilíbrio de adsorção foi alcançado dentro de $30 \mathrm{~min}$ para as condições estudadas e o mecanismo de adsorção empregando o modelo de Webber-Morris mostrou que a difusão intraporo predomina para tempos $<1$ minuto. O modelo de Boyd mostrou que o mecanismo muda para difusão 
intrafilme em $t>1$ minuto. A equação de Elovich mostrou que o processo de adsorção envolveu uma quimissorção. A análise dos modelos cinéticos pelo método não-linear mostrou que os dados experimentais foram melhor descritos pelo modelo de Pseudosegunda ordem com menor valor da função erro qui-quadrado. Os resultados mostraram que o adsorvente CCFP pode ser empregado para a remoção de $\mathrm{Pb}(\mathrm{II})$ de efluentes industriais.

\section{Agradecimentos}

Ao Conselho Nacional de Desenvolvimento Científico e Tecnológico (CNPq), a Fundação Cearense de Apoio ao Desenvolvimento Científico e Tecnológico (FUNCAP), ao Programa de Bolsas de Iniciação Científica da UECE (IC/UECE) e aos membros do Grupo de Pesquisa de Bioadsorção do CECITEC - UECE (GPBIO), a Universidade Estadual do Ceará.

\section{Referências Bibliográficas}

1 Jang, H.M., Yoo, S., Choi, Y-K, Park, S., Kan, E. Adsorption isotherm, kinetic modeling and mechanism of tetracycline on Pinus taedaderived activated biochar. Bioresource Technology 2018, 259, 24. [Link]

${ }^{2}$ Coelho, G.; Gonçalves, A. C.; Sousa, R. F. B.; Schwantes, D.; Miola, A. J.; Domingues, C. V. R. Uso de técnicas de adsorção utilizando resíduos agroindustriais na remoção de contaminantes em águas. Journal of Agronomic Sciences 2014, 27, 3. [Link]

${ }^{3}$ Departamento Nacional de Produção Mineral (DNPM) - Ministérios das Minas e Energia. [Link]

${ }^{4}$ Mavropoulos, E.; Dissertação de Mestrado, Fundação Oswaldo Cruz, 1999. [Link]

${ }^{5}$ Santos, I. C. DE A.; Monteiro, L. P. C.; Mainier, F. B. Tratamento de efluentes aquosos contendo cádmio, chumbo e cobre, a partir da técnica de eletrodeposição. Augustus 2015, 12, 40. [CrossRef]

${ }^{6}$ Bjorklund, K.; Li, L. Y. Adsorption of organic stormwater pollutants onto activated carbon from sewage sludge. Journal of Environmnental Managemet 2017, 7, [PubMed].

${ }^{7}$ Nossol, A. B. S.; Rosa, S. M. C.; Nossol, E.; Zarbin a. J. G.; Zamora P. P. Degradação fotocatalítica de corante utilizando-se nanocompósito $\mathrm{tio}_{2} /$ óxido de grafeno. Quimica Nova 2016, 5, 39. [CrossRef]

${ }^{8}$ Araújo, K. S.; Antonelli, R.; Gaydeczka, B.; Granato, A. C.; Malpass, G. R. P. Processos oxidativos avançados: uma revisão de fundamentos e aplicações no tratamento de águas residuais urbanas e efluentes industriais. Interdisciplinary Journal of Applied Science 2016, 14. 11. [CrossRef]

$9{ }^{9}$ Cerqueira, A. A.; Souza, P. S. A.; Souza, D. B.; Marques, M. R. C. Tratamento Eletrolítico da Água de Produção da Indústria de Petróleo: Sustentabilidade Ambiental e Pensamento Complexo. Revista Virtual de Química. 2014, 8, 6. [CrossRef]

${ }^{10}$ Bueno, M. Z.; Coral, L. A.; Sens, M. L.; Lapolli, F. R. Avaliação da nanofiltração e da osmose inversa na remoção de carbofurano em águas de abastecimento. Engenharia Sanitaria $e$ Ambiental 2016, 11, 447. [CrossRef]

${ }^{11}$ Zhang, H.; Gua, L.; Zhanga, L.; Zhenga, S.; Wana, H.; Suna, J.; Zhub, D.; Xua, Z. Removal of aqueous $\mathrm{Pb}(\mathrm{II})$ by adsorption on $\mathrm{Al}_{2} \mathrm{O}_{3}$ pillared layered $\mathrm{MnO}_{2}$. Applied Surface Science 2017, 8, 406. [CrossRef]

$12{ }^{12}$ Khan, N.A., Hasan, Z., Jhung, S.H., Adsorptive removal of hazardous materials using metal-organic frameworks (MOFs): A review. Journal of Hazardous Materials 2013, 244-245, 444. [CrossRef]

${ }^{13}$ Kanojia, A.; Jain, S. K. Performance of coconut shell as coarse aggregate in concrete. Construction and Building Materials 2017, 6, 150. [CrossRef]

${ }^{14}$ Vidil, Lisa.; Bilba, K.; Onésippe, C.; Arsène, M.; Savastano, H. Thermal insulating particle 
boards reinforced with coconut leaf sheaths. Green Materials 2016, 9, 4. [CrossRef]

${ }^{15}$ Sousa Neto, V. O. Tese de doutorado. Universidade Federal do Ceará, 2012. [Link]

${ }^{16}$ Noeline, B. F.; Manohar, D. M.; Anirudhan, T. S. Kinetic and equilibrium modelling of lead(II) sorption from water and wastewater by polymerized banana stem in a batch reactor. Separation and Purification Technology 2005, 9, 45. [CrossRef]

${ }^{17}$ Al-Degs, Y.; Khraisheh, M. A. M.; Allen S. J.; Ahmad, M. N. Effect of carbon surface chemistry on the removal of reactive dyes from textile effluent. Waters 2000, 8, 927. [CrossRef]

${ }^{18}$ Langmuir, J. The adsorption of gases on planes of glassmica and platinium. Journal of the American Chemical Society 1918, 42, 40. [CrossRef]

${ }^{19}$ Barrow, N. J. The description of phosphate adsorption curves. European Journal of Soil Science 1978, 15, 29. [CrossRef]

${ }^{20}$ Febrianto, J.; Kosasih, A. N.; Sunarso, J.; Indraswati, Y-H-J. N.; Ismadji, S.; Equilibrium and kinetic studies in adsorption of heavy metals using biosorbent: A summary of recent studies. Journal of Hazardous Materials 2009, 29, 162. [CrossRef]

${ }^{21}$ Masel, R. I.; Principles of adsorption and reaction on solid surfaces, 1st ed., John Wiley \& Sons Inc.: New York, 1996.
22 Malik, P.K. Use of activated carbons prepared from sawdust and rice-husk for adsorption of acid dyes: a case study of Acid Yellow. Dyes and Pigments 2003, 3, 56. [CrosRef]

${ }^{23}$ Ho, Y. S.; Makay, G. Sorption of Dye from Aqueous Solution by Peat. Chemical Engineering Journal 1998, 9, 70. [CrossRef]

${ }^{24}$ Rudzinsk, W.; Everett. D. H.; Adsorption of gases on heterogeneous surfaces, Academic Press: London, 1992. [Link]

${ }^{25}$ Yang, X.; Al-duri, B. Kinetic modeling of liquid-phase adsorption of reactive dyes on activated carbon. Journal of Colloid and Interface Science 2005, 9, 287. [CrossRef] [PubMed]

${ }^{26}$ Zeferino, L. F.; Ivanise, G. M. E.; Suárez-Iha, V.; Patricia, A. M. F. Adsorption of 5.5'Disulfonicindigotin (5.5'-DI) onto Green Coconut Fiber (Cocos nucifera L.): Kinetic and Isotherms. Journal of Encapsulation and Adsorption Sciences 2014, 2, 4. [CrossRef]

27 Boyd, G. E.; Schubert, J.; Adamson, A. W. The exchange adsorption of ions from aqueous solutions by organic zeolites, I: Ion exchange equilibria, Journal of the American Chemical Society 1947, 69, 2836. [CrossRef]

${ }^{28}$ Reichenberg, D. Properties of ion exchange resins in relation to their structure. III. Kinetics of exchange. Journal of the American Chemical Society. 1953, 8, 75. [CrossRef] 